Relmecs, diciembre 2018, vol. 8, no. 1, e032, ISSN 1853-7863

Universidad Nacional de La Plata. Facultad de Humanidades y Ciencias de la Educación.

Centro Interdisciplinario de Metodología de las Ciencias Sociales.

Red Latinoamericana de Metodología de las Ciencias Sociales

\title{
Un comienzo especial
}

\author{
Gabriela Rubilar * \\ *Universidad de Chile, Chile
}

Este primer número del año 2018 nos encuentra cargados de energía y con perspectivas muy positivas para este año que trae entre sus actividades la realización del VI Encuentro Latinoamericano de Metodología de las Ciencias Sociales (ELMeCS), el 7 al 9 de noviembre en la ciudad de Cuenca, Ecuador (http://elmecs.fahce.unlp.edu.ar/ y http://elmecs.fahce.unlp.edu.ar/ponencias). Este encuentro se organiza en torno a los ejes de la Innovación y creatividad en la investigación social, y han sido precisamente estas dimensiones, las que me he propuesto activar al momento de presentarles el $8 .^{\circ}$ volumen de la Revista ReLMeCS.

Los seis artículos que forman parte de este número son variados y heterogéneos en tus temas y aproximaciones, pero cuando los analizamos a partir de las nociones anteriores, estamos obligados a preguntarnos cómo la complejidad de la realidad social latinoamericana puede ser estudiada a través de una variedad de dispositivos, instrumentos y aproximaciones metodológicas que buscan analizarla desde las tensiones y contradicciones que caracterizan a los fenómenos sociales.

Los problemas complejos, ya abordados en la presentación del número anterior, a partir de las aportaciones de Edgar Morin (2004), demandan aproximaciones metodológicas innovadoras, pero especialmente creativas que desafían tanto a equipos de investigación como a los participantes en ellas.

Cómo nuestra investigación interviene y transforma los cuerpos de los investigadores y los fenómenos que investigamos, incluidos los propios sujetos de la investigación, es uno de los interrogantes que ha quedado latente tras la lectura de los artículos que forman parte de este volumen y que se constituye en un nuevo eje de análisis para analizar la innovación y la creatividad metodológica.

Esa creatividad metodológica supone un ejercicio explícito de reflexividad, que interroga constantemente nuestro quehacer, sometiéndolo a revisión y análisis, y que se encuentra muy bien plasmada en la reseña del libro ¿Condenados a la Reflexividad? Apuntes para repensar el proceso de investigación social, elaborada por Santiago García y Pedro Porta, quienes reconocen los aportes de la reflexividad al momento de pensar y (re)pensar los procesos de investigación social, analizando sus alcances, límites y posibilidades.

Tomando en cuenta esta invitación, en esta presentación del $8 .^{\circ}$ volumen de la revista, he querido analizar cómo los investigadores, que publican aquí los resultados de sus trabajos, desarrollan sus investigaciones, qué opciones

Cita sugerida: Rubilar, G. (2018). Un comienzo especial. Revista Latinoamericana de Metodología de las Ciencias Sociales, 8 (1), e032. https://doi.org/10.24215/18537863e032 
y derroteros metodológicos han seguido al momento de abordar ciertas cuestiones de interés investigativo y sobre todo qué decisiones toman al momento de comunicar y reportar sus resultados: ¿Qué elementos son comunes? ¿Qué constituye innovaciones? Esas son algunas de las interrogantes que se activan en la lectura de los artículos que forman parte de este volumen, encontrando, incluso, que quienes analizan temáticas similares lo hacen desde perspectivas teóricas y metodológicas distintas, lo que da cuenta del potencial innovador y la creatividad que se puede desplegar en las líneas de trabajo de cada autor o equipo de investigación.

Es así como nos encontramos con el artículo de Agustín Arakaki, "Revisión de las metodologías de estimación de la pobreza en Argentina. Aportes para el debate”. Agustín se atreve a ir un poco más allá de los debates sobre ajustes metodológicos y discrepancias entre las mediciones oficiales y las alternativas de la pobreza en Argentina, proponiendo un cambio en la metodología de medición oficial. Sus "innovaciones" suponen salir del área de confort o comodidad de aquello que se hace de manera estandarizada, usando fuentes alternativas que aportan mayor confiabilidad a los resultados. Paradójicamente, su propuesta de ajuste introduce incertidumbre a la metodología oficial, y sus resultados le permiten explorar otras posibilidades metodológicas, cuyos alcances suponen implicancias de política. Una incomodidad que el autor explora y recoge en su revisión histórica, analizando los inconvenientes y dificultades de los distintos aspectos involucrados con la medición, pero que al mismo tiempo le obligan a asumir un posicionamiento metodológico al momento de sugerir caminos de salidas. Innovar en pobreza implica también innovar en las formas de medición y en el uso que se da a sus resultados.

Los usos de las mediciones oficiales también se encuentran contenidos en el artículo de María Marcela Crovetto, Mercedes Ejarque y María Virginia Nessi, "El trabajo infantil y adolescente del agro argentino: consideraciones sobre su medición en las estadísticas públicas y una propuesta alternativa”. Las autoras, siguiendo un esquema que encuentra conexiones con el artículo anterior, proponen y aplican un camino metodológico mixto que cuestiona los presupuestos sobre los que se construye la información acerca el trabajo infantil y adolescente en la Argentina, introduciendo innovaciones que permitan capturar mejor su complejidad y dinamicidad. La propuesta construida no solo resulta una solución creativa a los problemas de subregistro y sus vacíos de información, sino que supone desarrollar formas innovadoras para abordar las limitaciones y proponer mecanismos de construcción de información pertinentes a los sujetos y problemáticas de estudio. Esto resulta especialmente relevante ya que el trabajo infantil y adolescente suele quedar oscurecido por las dinámicas propias del mercado agropecuario y sus lógicas de funcionamiento, elementos que también se encuentran presentes en otras dinámicas de mercado de trabajo que tienen expresiones locales. "Visibilidad y voz" son los alcances de esta propuesta metodológica que introduce una alteración en los sistemas de registro, haciéndolos sensibles a los marcos internacionales que se han construido en este ámbito (OIT, UNICEF), al mismo tiempo que reconocen la existencia de "aliados estratégicos locales" que abogan por el reconocimiento de situaciones que constituyen puntos ciegos de los sistemas de medición y de sus resultados en términos de políticas públicas.

Las propuestas de medición y sus consecuentes debates metodológicos también se encuentran presentes en el artículo de Mariana Marcos, María Mercedes Di Virgilio y Gabriela Mera. En su escrito, "El déficit habitacional en Argentina. Una propuesta de medición para establecer magnitudes, tipos y áreas prioritarias de intervención intra-urbana”, estas investigadoras realizan una aproximación que se ubica en los entrecruces de distintas disciplinas, con el fin de abordar los problemas de déficit de vivienda que afectan a las ciudades latinoamericanas. La propuesta de análisis microespacial adquiere sentido en el marco de los estudios urbanos y de los análisis de la dimensión espacial, ya que posee aplicaciones no sólo para las decisiones de política de vivienda y sus efectos sobre el territorio en diferentes escalas territoriales (barrial, local, metropolitana). Aquí cobra sentido una propuesta que analice el déficit de vivienda en relación con variables macro-meso y micro que inciden en los patrones de calidad de vida, como los sistemas de transporte público, el acceso a servicio y el equipamiento comunitario. Salcedo en su trabajo sobre los "mitos de las ciudades” ofrece una interesante discusión para analizar y discutir creativamente algunos de los resultados de esta investigación y los supuestos sobre los que abordar el déficit habitacional hoy. Invito a leer este artículo a la luz de algunos debates contemporáneos sobe el uso del espacio urbano y déficit habitacional, al mismo tiempo que se reflexiona activamente acerca de la ciudad. Así lo indica Salcedo, cuando recurre a los aportes de Michel de Certau para afirmar que "el espacio social habitado es el 
resultado de un conflicto permanente entre poder y resistencia al poder” (2017, p. 66).

Las relaciones, distancias y posicionamientos entre maestros y discípulos, aparecen muy bien ilustradas en el primer artículo de este volumen. Gabriel Nardacchione y Pablo Tovillas analizan explícitamente las controversias entre Pierre Bourdieu y Luc Boltanski, en torno al problema de la acción y la axiología del investigador. Los investigadores analizan las continuidades y discontinuidades en los planteamientos y pensamientos de ambos autores, con énfasis en el problema de la ética política y la dimensión axiológica del trabajo del sociólogo. Son precisamente estas dimensiones de análisis las que hemos querido poner de relieve en esta presentación, que observa la innovación y la creatividad en los procesos de investigación, al mismo tiempo que se interroga por las tradiciones. Ruptura y continuidad son las claves analíticas que los autores usan para comprender el posicionamiento de Bourdieu y Boltanski ante el problema de la acción y el modo como ambos autores desarrollan un posicionamiento al respecto. Posición que, en clave de esta presentación, interpela a la posición teórica/epistemológica de estos dos autores y que tiene influencia en la generación de "escuelas", discípulos y seguidores. Observamos en este artículo cómo de la mano de los maestros se forja el interés investigativo, se interroga el propio quehacer, al mismo tiempo que se potencia la creatividad y la construcción de nuevas posibilidades investigativas, nuevos abordajes conceptuales y nuevas posibilidades paradigmáticas para sustentar y repensar el quehacer investigativo.

Algunas aproximaciones teóricas y epistemológicas similares a la idea de implicación, acción y transformación del primer artículo, se encuentran contenidas en el artículo de Seín Laparra "Pensamiento indígena y construcción del conocimiento en educación. Hacia una propuesta de implicación pedagógica en la formación docente intercultural”. En este trabajo se siguen algunas discusiones en torno a socio-epistemología y se propone un modelo de investigación de siete dimensiones que transforma las maneras en que se concibe y piensa la investigación. Uno de los aspectos de este modelo promueve el paso de un pensamiento tridimensional (trinitario) a uno tetradimensional, que cambia radicalmente las formas de aproximación a las preguntas/problemas de investigación y que vuelve a los investigadores responsables de los resultados/alcances/efectos de sus investigaciones. La acción de pensar y de investigar no es concebida desde el pensamiento indígena sólo como un acto racional, sino que implica también una dimensión política, estética y ética de pensar la investigación. Lo que proporciona una clave onto-epistemológica distinta para analizar el conocimiento que se construye, otorgándole una posibilidad de trascendencia que va más allá de los marcos tradicionales.

Finalmente, Gabriel Levita en su artículo “¿Qué pretende usted de mí? Enfrentando obstáculos en las entrevistas a elites en una investigación sobre los senadores nacionales argentinos” pone nuevamente de relieve la cuestión de las entrevistas a las elites y los alcances de este tipo de abordajes en materia de investigación. Resulta imposible no vincular este artículo con las reflexiones clásicas de Dexter (1970) acerca del sentido de los informantes claves y el modo en que las entrevistas a actores especializados tensionan y desafían metodológicamente nuestros trabajos. La pregunta ¿qué pretende usted de mí?, formulada por los investigadores y analizada en clave biográfica por el autor de este artículo, nos lleva a preguntarnos explícitamente qué pretendemos con nuestra investigación y cuáles son sus alcances. Reflexividad investigadora, responsabilización e incidencia públicas son los elementos que se encuentran en la base de este artículo, cuyos contenidos nos invitan a repensar las estrategias metodológicas y los alcances prácticos de nuestro quehacer investigativo.

Los artículos que presentamos en este volumen suponen un trabajo de investigación "bien hecho", con estándares de calidad que se encuentran presentes en todas las fases del proceso de investigación, incluida su trastienda (Wainerman y Sautu, 1997; Castillo, Valles y Wainerman, 2009), y con desarrollo de un espíritu creativo y artístico, en el sentido de artesanía intelectual (Mills, 1994, donde la calidad de la investigación no está determinada por la complejidad técnica con que se abarca la temática sino por la forma en que se asume la responsabilidad y el aprendizaje de su práctica. 


\section{Bibliografía}

Castillo, J.J.; Valles, M. y Waineman, C. (ed.) (2009) La trastienda de la investigación social. Teoría e investigación concreta en primera persona. Revista Política y Sociedad, 46(3). Número especial.

Dexter, L. A. (1970). Elite and specialized interviewing. Evanston: Northwestern University Press.

Mills, C. W. (1994). La imaginación sociológica Buenos Aires: FCE.

Morin, E. (2004). La epistemología de la complejidad. Gazeta de antropología, 20(2), 1-14.

Salcedo, R. (2018) “Cinco mitos sobre las ciudades” en Salcedo (In memorian). Santiago de Chile: Editorial Bifurcaciones

Wainerman, C. y Sautu, R. (1997) La trastienda de la investigación. Buenos Aires: Lumiere. 